\title{
Next-generation sequencing diagnostics of bacteremia in septic patients
}

Silke Grumaz ${ }^{1 \dagger}$, Philip Stevens ${ }^{2,4 \dagger}$, Christian Grumaz', Sebastian O. Decker ${ }^{3}$, Markus A. Weigand ${ }^{3}$, Stefan Hofer ${ }^{3}$, Thorsten Brenner ${ }^{3}$, Arndt von Haeseler ${ }^{4,5}$ and Kai Sohn ${ }^{1,2^{*}}$

\begin{abstract}
Background: Bloodstream infections remain one of the major challenges in intensive care units, leading to sepsis or even septic shock in many cases. Due to the lack of timely diagnostic approaches with sufficient sensitivity, mortality rates of sepsis are still unacceptably high. However a prompt diagnosis of the causative microorganism is critical to significantly improve outcome of bloodstream infections. Although various targeted molecular tests for blood samples are available, time-consuming blood culture-based approaches still represent the standard of care for the identification of bacteria.
\end{abstract}

Methods: Here we describe the establishment of a complete diagnostic workflow for the identification of infectious microorganisms from seven septic patients based on unbiased sequence analyses of free circulating DNA from plasma by next-generation sequencing.

Results: We found significant levels of DNA fragments derived from pathogenic bacteria in samples from septic patients. Quantitative evaluation of normalized read counts and introduction of a sepsis indicating quantifier (SIQ) score allowed for an unambiguous identification of Gram-positive as well as Gram-negative bacteria that exactly matched with blood cultures from corresponding patient samples. In addition, we also identified species from samples where blood cultures were negative. Reads of non-human origin also comprised fragments derived from antimicrobial resistance genes, showing that, in principle, prediction of specific types of resistance might be possible.

Conclusions: The complete workflow from sample preparation to species identification report could be accomplished in roughly $30 \mathrm{~h}$, thus making this approach a promising diagnostic platform for critically ill patients suffering from bloodstream infections.

Keywords: Sepsis, Diagnostics, Next-generation sequencing, Circulating nucleic acids

\section{Background}

Sepsis remains a challenge in intensive care medicine, its incidence increasing continuously over the past decades $[1,2]$. Despite massive efforts in sepsis research, new therapeutic approaches are rare and mortality in patients with septic shock still remains unacceptably high $[1,2]$. In addition to an early focus on control, recent guidelines recommend the initiation of an empiric antibiotic therapy as early as possible (preferably within $1 \mathrm{~h}$ ) following diagnosis of sepsis [3]. However, the identification of

\footnotetext{
* Correspondence: kai.sohn@igb.fraunhofer.de

${ }^{\dagger}$ Equal contributors

${ }^{1}$ Fraunhofer IGB, Nobelstr. 12, 70569 Stuttgart, Germany

${ }^{2}$ IGVP, University of Stuttgart, Nobelstr. 12, 70569 Stuttgart, Germany

Full list of author information is available at the end of the article
}

the causative pathogen as well as its resistance pattern is crucial for early optimization of the antimicrobial treatment regime. In this context, culture-based diagnostic procedures (e.g., blood cultures) represent the standard of care, although they are associated with relevant limitations $[3,4]$ : (i) depending on microbiological growth, it often takes up to 5 days for results to become available; and (ii) culture-based diagnostic procedures often reveal false negative results due to the administration of an empiric antibiotic therapy. Accordingly, patients suffering from septic disease are at high risk for antimicrobial overtreatment, antibiotics-related toxicity, and the selection of multi-drug resistant pathogens due to the inadequate and prolonged use of broad spectrum antibiotics. In this 
context, culture-independent molecular diagnostic procedures (e.g., PCR-based techniques) have already been introduced for the identification of the causative pathogen in infected patients [5-9]. However, the occurrence of ambiguous results as well as limitations in the quantitative measurement of the bacterial load in patients' samples and detection of antibiotic resistance markers are known limitations of these PCR-based diagnostic approaches. Therefore, the concept of our work was to develop an alternative diagnostic platform for the identification of infectious microorganisms based on unbiased sequence analyses of circulating cell-free DNA (cfDNA) from plasma samples of septic patients by next-generation sequencing (NGS). In addition, we demonstrate the applicability of NGS for the detection of antibiotic resistance markers in plasma.

\section{Methods}

\section{Study design}

Human data are from a secondary analysis of a subset of patients participating in the RAMMSES trial (German Clinical Trials Register, DRKS00000505) [10]. An amendment to the existing approval (TrialCode-Nr. S123-2009) was written and approved by the local ethics committee.

The observational clinical study was conducted in the surgical intensive care unit (ICU) of Heidelberg University Hospital, Germany. Study and control patients or their legal designees signed a written informed consent. In total, 120 patients in three groups were consecutively enrolled into the study from August 2009 to July 2010. The three groups included: (1) 60 patients with septic shock according to the criteria of the International Sepsis Definitions Conference [11] and due to documented or suspected infection according to the criteria of the International Sepsis Forum Consensus Conference on Definitions of Infection in the Intensive Care Unit [12]; (2) 30 postoperative controls following major abdominal surgery without any evidence of infection; and (3) 30 healthy volunteers. Plasma samples from patients with septic shock were collected at sepsis onset (T0) and $24 \mathrm{~h}$ (T1), 4 days (T2), 7 days (T3), 14 days (T4), and 28 days (T5) later. Plasma samples from the postoperative group were collected prior to surgery (T0), immediately following the end of the surgical procedure (T1), and 24 h later (T2). Plasma samples from the volunteer group were collected once (T0).

For this secondary analysis, patients' electronic medical records were retrospectively screened for results from blood culture testing during septicemia and patients were selected according to availability of microbiological data for similar time points, which led to the smaller sample size within this proof-ofconcept study.

\section{Clinical microbiology}

Blood culture testing in Heidelberg University Hospital is routinely performed as described [13]. Whole blood samples are obtained via direct venipuncture (e.g., antecubital vein) applying sterile techniques and $10 \mathrm{~mL}$ blood is inoculated to both an aerobic and an anaerobic liquid culture medium (BACTEC PLUS, BD Biosciences, Heidelberg, Germany). Cultures are incubated for 5 days (BACTEC, BD Biosciences, Heidelberg, Germany) and positive cultures are analyzed according to approved inhouse hospital standard techniques, including identification by VITEK2 (Biomerieux, Nuertingen, Germany) or MALDI TOF (Bruker, Madison, WI, USA) and automated antimicrobial susceptibility testing (VITEK 2). Quantification of HSV1 DNA and cytomegalovirus DNA from plasma or tracheal secretion was performed via quantitative real time PCR as previously described [14]. Cultivation of wound swabs and catheter and stool samples was carried out as previously described $[15,16]$.

\section{Plasma preparation and nucleic acid isolation}

Plasma was prepared from blood samples by centrifugation for $10 \mathrm{~min}$ at $292 \times \mathrm{g}$ and $4{ }^{\circ} \mathrm{C}$, snap frozen, and stored at $-80{ }^{\circ} \mathrm{C}$ until further processing. Nucleic acids were isolated from thawed plasma after a centrifugation step of $5 \mathrm{~min}$ at $1000 \times \mathrm{g}$ with the Circulating Nucleic Acid Kit (Qiagen) according to the manufacturer's protocol with the following exceptions: plasma volumes after centrifugation (from 130 to $790 \mu \mathrm{l}$ ) were adjusted to $1 \mathrm{ml}$ with sterile phosphate buffered saline. Final elution of the nucleic acids from the spin column was carried out with $30 \mu \mathrm{l}$ molecular biology grade water (5 Prime, Germany). Contamination controls were prepared following the same procedure, starting from $1 \mathrm{ml}$ molecular biology grade water (5 Prime, Germany) and $1 \mathrm{ml}$ of sterile phosphate buffered saline, which was prepared using the Circulating Nucleic Acid Kit (Qiagen). The cfDNA was quantified with the Qubit dsDNA HS Assay Kit (Life Technologies) and quality was assessed by the High Sensitivity DNA Kit on a Bioanalyzer (Agilent).

\section{Preparation of NGS libraries and sequencing}

Libraries for NGS were prepared from 1 ng cfDNA using the Nextera XT library preparation Kit (Illumina) according to the manufacturer's protocol, with the exception that the final elution after bead clean-up was carried out in $34 \mu \mathrm{l}$ of resuspension buffer (Illumina). A further contamination control was added by using $5 \mu \mathrm{l}$ of molecular biology grade water (5 Prime, Germany) as template for the Nextera XT Library Preparation Kit (Illumina). Sequencing of the libraries was performed on a HiSeq2500 (Illumina), resulting in 25-30 million 100-bp single end reads, on average, per sample. Since samples V6, V22, and P6 were initially sequenced with considerably 
higher numbers of reads, those samples were randomly reduced in silico to 30 million representative subsampled reads.

\section{Bioinformatics}

Raw reads were cleared from potential adapter contamination, quality controlled, and, if necessary, trimmed using BBDuk (https://sourceforge.net/projects/bbmap/). To pass the quality filter, read quality needed to surpass a Phred score of 20 and achieve a minimal length of $50 \mathrm{bp}$ after trimming of low quality and adapter bases. Subsequently, NextGenMap was used to align qualitycontrolled reads to the human reference genome (hg19) requiring a minimum identity between read and reference genome of $80 \%$. Reads mapping to the human reference genome and reads with low complexity (consecutive stretches of di- and trinucleotides along the whole read sequence) were excluded from further analysis [17]. Finally, Kraken was used to assign reads to systematic classification using the RefSeq database (release version 68) comprising 35,749 bacterial and 4340 viral genomes complemented by 12 selected fungal genomes (Additional file 1: Table S1). Since several Xanthomonas species are described as well-known contaminants, we excluded Xanthomonas reads as well as the Illumina sequencing spike-in PhiX [18].

To quantitatively compare the number of reads that map to different microbial taxonomic classifications between different samples, we normalized the read counts by the respective library size (Table 1$)$.

We introduced the $n \times(s+1)$ dimensional count matrix $\mathrm{D}$, where $n$ is the number of control samples and $s$ the number of species detected in all samples. Thus, $D_{i j}$ defines the number of reads found in control sample $i$ for species $j . D_{i,(s+1)}$ defines the number of reads which cannot be assigned to any species. One notes that $D_{i,(s+1)}$ is usually larger than the $D_{i j}$ s. Then Eq. 1 is the maximum likelihood estimate of the probability to observe species $j$ in a control sample:

$$
\hat{p}_{j}=\frac{\sum_{k=1}^{n} D_{k, j}}{\sum_{k=1}^{n} D_{k, s+1}}, j=1, \ldots, s+1
$$

Since the number of reads for one species is typically low, we assumed that the read counts for species $j$ are Poisson distributed with parameter:

$$
\lambda_{j}=\frac{\sum_{k=1}^{n} D_{k, j}}{n} .
$$

To test this assumption for each species a standard $\chi^{2}$ goodness of fit test is performed.

For reads sequenced from patient plasma, the same data processing pipeline is applied, which yields a read count vector $C=\left(C_{1}, \ldots, C_{s}, C_{s}+1\right)$. Based on the Poisson distribution with species-specific parameter $\lambda_{j}$, the $p$ value to observe at least $C_{j}$ read counts in a patient sample is computed as:

$$
P\left(X \geq C_{j} \mid \lambda_{j}\right)=\sum_{k \geq C_{j}} \frac{e^{-\lambda_{j}} \lambda_{j}^{k}}{k !} .
$$

If this $p$ value is small, then one would reject the hypothesis that the read count of species $j$ in the patient sample follows the Poisson distribution derived from the healthy individuals and conclude that the respective species occurs too often in the patient.

Now, with the given species specific $\lambda$ we can compute the sepsis indicating quantifier (SIQ) score as follows:

$$
S I Q_{j}=C_{j} *-\left(\log _{10}\left(P\left(X \geq C_{j} \mid \lambda_{j}\right)\right)\right)
$$

The SIQ score now gives rise to a quantitative and probabilistic assessment of every detected microbe in the respective sample.

In order to identify resistance genes, potential microbial reads were mapped against the downloaded CARD resistance gene database using NextGenMap with the following parameters: sensitivity $(-\mathrm{s})$ and minimal number of mapped residues (-R) of 0.9 . We required $100 \%$ identity between read sequence and target sequence in the CARD database.

\section{Results}

\section{Elevated levels of cfDNA in septic patients reveal microbial DNA fragments}

In order to test the diagnostic potential of cfDNA to identify infecting microorganisms in septic patients, in total 62 plasma samples from septic patients $(S, n=7)$, healthy volunteers $(\mathrm{V}, n=12)$, and patients that underwent abdominal surgery $(P, n=6)$ at different time points were analyzed in this study. Mean ages were 70.5 years, 26.4 years, and 64.2 years for groups $\mathrm{S}, \mathrm{V}$, and P, respectively (Table 1 ). Septic patients were monitored by a comprehensive clinical microbiology diagnostic workup. cfDNA isolation from patient and control plasma revealed a characteristic, predominantly apoptosis-associated size pattern $[19,20]$ (Additional file 2: Figure S1). Due to the small cohort size, it was not possible to draw statistically relevant conclusions; however, concentrations of cfDNA tend to be increased in patients with septic shock (S, mean $197.23 \mathrm{ng} / \mathrm{ml}$ ), especially at the onset of sepsis (S T0, mean $377.30 \mathrm{ng} / \mathrm{ml}$ ) (Fig. 1), which is consistent with other reports [21, 22]. Elevated concentrations were also measured in plasma of post-surgery patients (P T1-T2, mean $451.63 \mathrm{ng} / \mathrm{ml}$ ) compared with uninfected controls before surgery and healthy volunteers ( $\mathrm{P}$ T0, mean $149.33 \mathrm{ng} / \mathrm{ml}$; V, mean 
$55.43 \mathrm{ng} / \mathrm{ml})$. cfDNA sequencing revealed a similar proportion of human reads for all groups with 96.36, 96.52, and $96.21 \%$ in groups S T0, V, and P T0, respectively (Table 1 ). The average classified reads were higher in septic patients at the onset of sepsis compared with the control groups (9.82, 3.50, and $2.64 \%$ in groups S T0, V, and P T0), indicating a higher bacterial load. It should be noted, however, that the proportion of classified reads includes bleed-through PhiX reads and reads classified as from Xanthomonas campestris, which were discarded as contaminants in downstream analysis.
Establishment of the SIQ score as a quantitative score for pathogen calling

Figure 2a summarizes the principle for diagnostic assessment of microbial reads for a patient in comparison with uninfected controls. Normalized read counts of classified, non-human reads for each plasma sample were calculated, resulting in a quantification of distinct species (Additional file 3: Table S6). In case of patient S9 with positive blood cultures for Enterobacter cloacae at the onset of sepsis, distinctly higher numbers of E. cloacae reads were found compared with a set of species with normalized reads at very low abundances (Fig. 2b).

Table 1 Patient characteristics, cfDNA concentration, and sequencing statistics

\begin{tabular}{|c|c|c|c|c|c|c|c|c|}
\hline ID & Time & Sex & Age (years) & cfDNA (ng/ml plasma) & Sequencing depth & Human reads (\%) & Unmapped (\%) & Classified (\%) \\
\hline S9 & TO & $M$ & 82 & 120.59 & $30,650,143$ & 92.90 & 7.10 & 28.90 \\
\hline S10 & TO & M & 68 & 307.83 & $27,199,593$ & 98.70 & 1.30 & 2.85 \\
\hline S11 & TO & M & 62 & 805.50 & $27,073,879$ & 93.61 & 6.39 & 20.73 \\
\hline S19 & TO & $\mathrm{F}$ & 62 & 101.30 & $26,892,684$ & 98.45 & 1.55 & 4.75 \\
\hline S23 & TO & M & 79 & 146.70 & $24,917,032$ & 97.12 & 2.88 & 3.85 \\
\hline S26 & T0 & M & 66 & 1088.90 & $32,529,889$ & 96.60 & 3.40 & 3.24 \\
\hline \multirow[t]{3}{*}{ S60 } & TO & $\mathrm{F}$ & 70 & 70.29 & $27,381,853$ & 97.10 & 2.90 & 4.40 \\
\hline & & Average S T0 & 70 & 377.30 & $28,092,153$ & 96.36 & 3.64 & 9.82 \\
\hline & & Average $\mathrm{S}$ all & 70 & 197.23 & $25,960,730$ & 97.79 & 2.21 & 4.24 \\
\hline V5 & & M & 24 & 35.80 & $34,203,815$ & 81.90 & 18.10 & 12.38 \\
\hline V6 & & M & 29 & 27.40 & $30,000,000$ & 98.96 & 1.04 & 2.25 \\
\hline V7 & & F & 22 & 76.40 & $21,004,601$ & 96.58 & 3.42 & 2.35 \\
\hline V13 & & F & 26 & 23.50 & $24,449,232$ & 98.09 & 1.91 & 3.26 \\
\hline V14 & & M & 28 & 38.60 & $37,971,559$ & 97.42 & 2.58 & 1.79 \\
\hline V15 & & M & 27 & 166.80 & $24,505,696$ & 97.60 & 2.40 & 2.88 \\
\hline V16 & & F & 29 & 70.60 & $27,220,925$ & 97.06 & 2.94 & 2.67 \\
\hline V17 & & M & 26 & 28.40 & $20,225,374$ & 98.61 & 1.39 & 3.30 \\
\hline V18 & & M & 28 & 48.80 & $19,157,938$ & 98.14 & 1.86 & 2.46 \\
\hline V19 & & F & 31 & 33.40 & $25,776,920$ & 97.08 & 2.92 & 2.87 \\
\hline V21 & & M & 22 & 67.30 & $25,220,391$ & 97.72 & 2.28 & 2.51 \\
\hline \multirow[t]{2}{*}{ V22 } & & M & 25 & 48.20 & $30,000,000$ & 99.15 & 0.85 & 3.25 \\
\hline & & Average V & 26 & 55.43 & $26,644,704$ & 96.52 & 3.48 & 3.50 \\
\hline P1 & T0 & M & 58 & 50.20 & $22,389,868$ & 96.95 & 3.05 & 1.79 \\
\hline P2 & TO & M & 53 & 552.00 & $30,000,000$ & 98.57 & 1.43 & 3.35 \\
\hline P3 & TO & F & 62 & 109.50 & $18,796,573$ & 94.69 & 5.31 & 1.38 \\
\hline P4 & T0 & $\mathrm{F}$ & 72 & 36.42 & $28,457,744$ & 94.91 & 5.09 & 4.59 \\
\hline P5 & T0 & M & 64 & 65.38 & $28,547,804$ & 95.48 & 4.52 & 3.72 \\
\hline \multirow[t]{4}{*}{ P7 } & TO & M & 76 & 82.50 & $28,845,398$ & 96.69 & 3.31 & 1.00 \\
\hline & & Average P T0 & 64 & 149.33 & $26,172,898$ & 96.21 & 3.79 & 2.64 \\
\hline & & Average P T1-T2 & 64 & 451.63 & $25,406,269$ & 97.72 & 2.28 & 2.14 \\
\hline & & Average $\mathrm{P}$ all & 64 & 350.86 & $25,661,812$ & 97.22 & 2.78 & 2.31 \\
\hline
\end{tabular}

Patients were grouped as septic patients $(S)$, healthy volunteers $(V)$, and non-infected patients following major abdominal surgery $(P)$. Of the total reads (sequencing depth), all reads mapped to human reference genome hg19 are classified as human reads; the remaining reads are denoted as unmapped. The proportion of unmapped reads classified to any species using Kraken are specified here as classified. $F$ female, $M$ male 
A

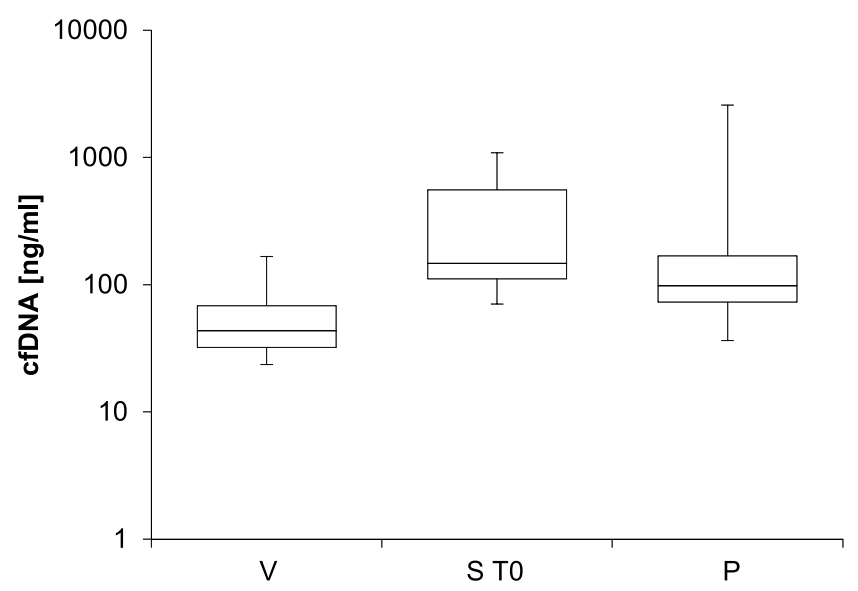

B

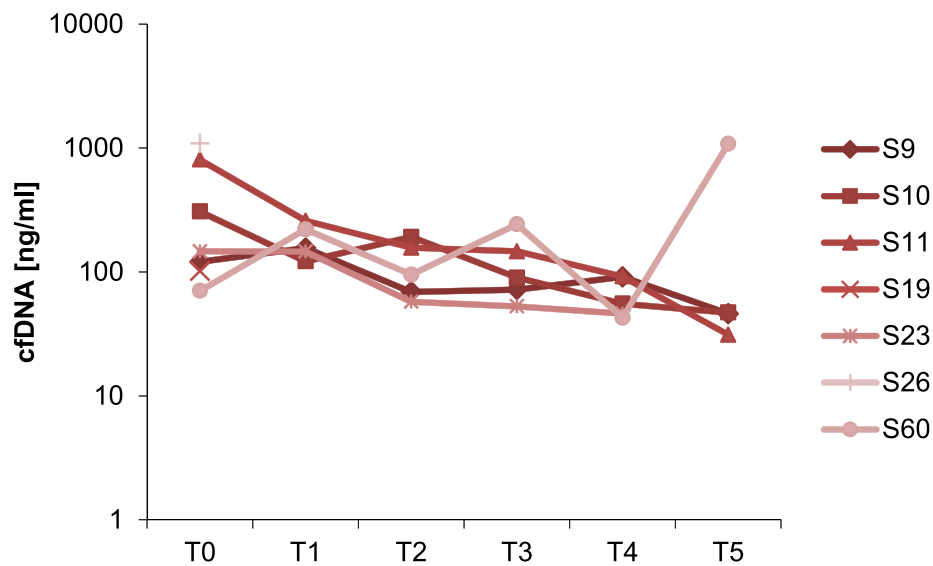

C

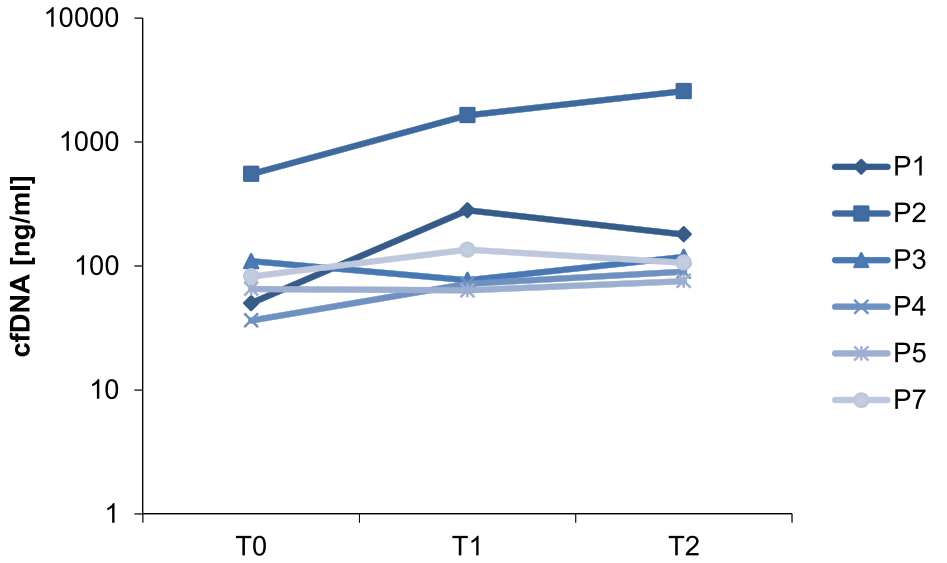

Fig. 1 Distribution of cfDNA concentrations over different patient groups and time points. a Comparison of cfDNA concentrations between healthy volunteers ( $V$, septic patients at the onset of sepsis (STO), and non-infected patients following major abdominal surgery (P). b Alterations in cfDNA concentrations of septic patients' plasma samples collected over the observational period of the trial. Samples were obtained at sepsis onset (T0), after $24 \mathrm{~h}$ (T1), 4 days (T2), 7 days (T3), 14 days (T4), and 28 days (T5). c Comparison of cfDNA concentrations in patients undergoing major abdominal surgery without evidence of infection. Blood samples from the postoperative group were collected prior to surgery (TO), immediately following the end of the surgical procedure (T1), and $24 \mathrm{~h}$ later (T2) 


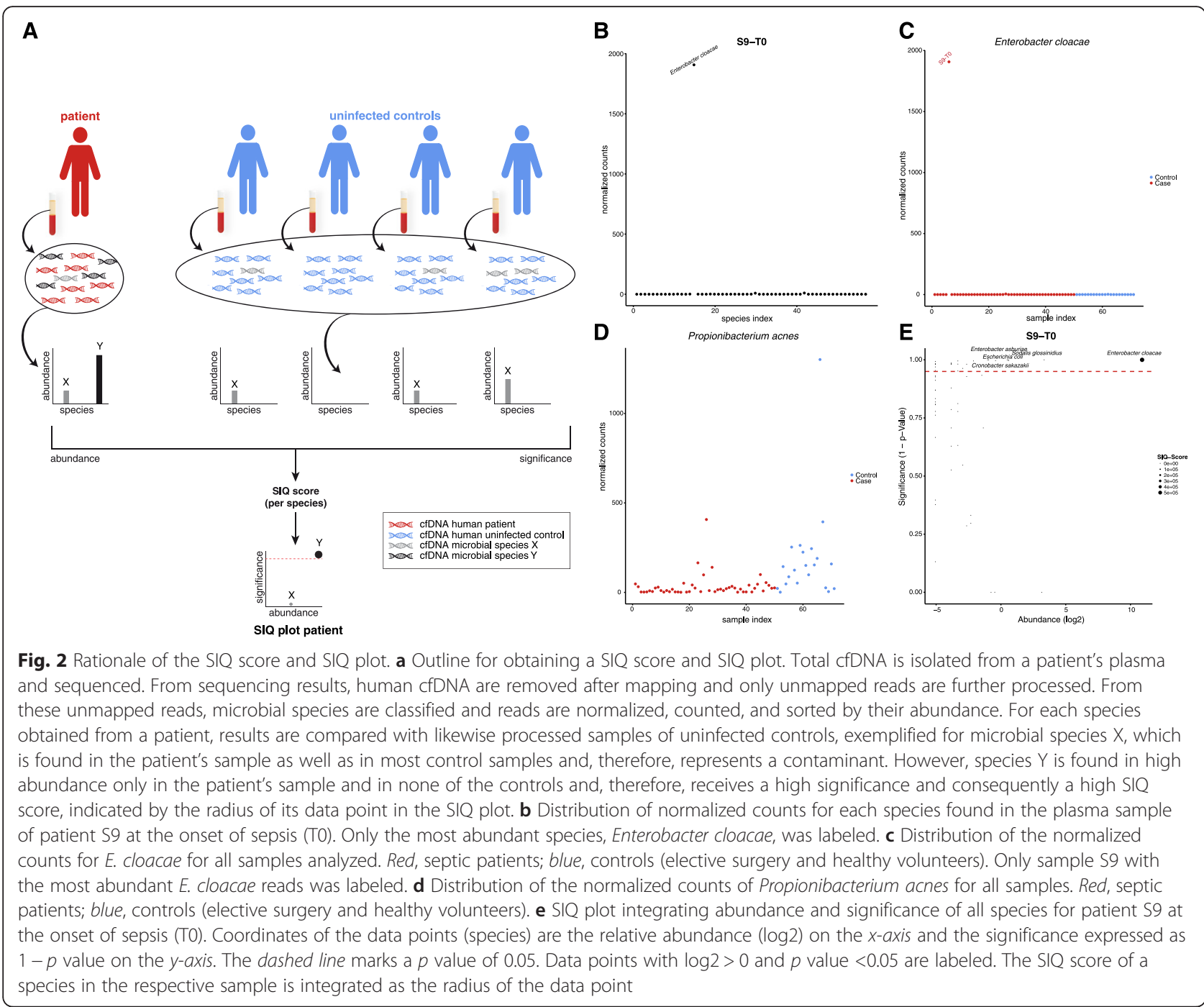

To evaluate the significance of read abundances for all species classified, normalized read counts were compared for each species between all septic patients and controls. The abundance of normalized reads for $E$. cloacae in patients and controls was close to zero except for time point T0 of patient S9 (1,907.33; Fig. 2c; Additional file 4: Figure S2). In contrast, normalized read counts for a common skin commensal like Propionibacterium acnes varied significantly between all samples and higher abundances were especially detected in samples obtained from uninfected controls (Fig. 2d; Additional file 5: Figure S3). This effect was observed for several bona fide contaminant species (Additional file 6: Table S2). To support discrimination by relevance, we combined abundance and unlikeliness of observed read counts for each species found in a sample. The $\log 2$ ratio of normalized reads is plotted versus $1-p$ value (Fig. 2e). Species which are significantly represented in a sample with a $p$ value $<0.05$ compared with controls are considered significant (Fig. 2e). An individual SIQ score was calculated as a product of abundance and significance and is indicated by the radius of the corresponding data point. Therefore, the species with highest relevance found in cfDNA of patient $S 9$ at the onset of sepsis was E. cloacae with a SIQ score of 586,795 (Additional file 7: Table S3). In contrast, no significant SIQ scores were observed for uninfected postoperative patients (Additional file 7: Table S3 and Additional file 8: Figure S4)

\section{Clinical relevance of the SIQ score}

The SIQ score can also be used for time-course monitoring of a patient's progression and response to targeted treatment. We therefore compared the SIQ score with clinical microbiology data, supplemented with data on anti-infective therapy (Additional file 9: Table S5) over a trial period of 28 days. Patient S10 developed septic shock due to severe pneumonia following a gastrectomy 
to remove a tumor of his stomach (Fig. 3a). On study inclusion, blood culture and subsequent samples from different sites were positive for Staphylococcus aureus. In addition to $S$. aureus, significant viral burden (herpes simplex virus 1 (HSV1); $9.87 \times 10^{6}$ copies $/ \mathrm{ml}$ ) and low fungal burden (Candida albicans) were also detected in other specimens by quantitative PCR (qPCR) or cultivation, respectively. Sequencing of plasma samples confirmed $S$. aureus as the predominant organism in the patient's blood at all observed time points with SIQ scores of 125.24, 720.41, 12.86, 2, and 0.03. Interestingly, the highest SIQ score for $S$. aureus was observed one day following inclusion and strongly declined afterwards. On day 7 the highest SIQ score was obtained for HSV1 (43.07), which was also detected by qPCR on day 14 in tracheal secretions $\left(8.5 \times 10^{5}\right.$ copies $\left./ \mathrm{ml}\right)$ but otherwise in no other sample (qPCR or NGS). Patient S60 (Fig. 3b) suffered from recurring episodes of bowel leakage accompanied by several septic hits and underwent repetitive reconstructive abdominal surgery. Remarkably, during the course of sepsis, no blood culture was positive for this patient, with the exception of a single bottle positive for Staphylococcus epidermidis one day before study inclusion. However, wound swabs and intraabdominal lavage were positive for Escherichia coli and Enterococcus faecium. Tracheal secretions furthermore tested positive for HSV1 and/or C. albicans. During the course of infection, the patient developed a ventilator-associated pneumonia which was due to E. coli, Stenotrophomonas maltophilia, and Klebsiella pneumoniae. In contrast to standard blood culture, SIQ score analyses of this patient's plasma were in good agreement with non-blood culture clinical microbiology results over the complete time course. Samples collected at the early septic phase (days 0 , $1,4,7,14$, and 21) confirmed the clinical representation of a polymicrobial abdominal infection due to E. coli, $E$. faecium, and Bacteroides fragilis. Furthermore, results from qPCR of HSV1 from tracheal secretions could be confirmed by NGS (day 7). The progression of the patient towards ventilator-associated pneumonia at the end of our study period was also confirmed by SIQ scores of 1341.37 for K. pneumonia and 68.58 for B. fragilis in plasma obtained on day 28. For additional patients NGS results from plasma samples matched data from other specimens, such as tracheal secretion, swabs, or catheter cultivation (Additional files 10, 11, and 12: Figures S5-S7).

\section{Identification of resistance genes}

Resistance to antibiotics is mediated by the expression of resistance genes, including mecA or $v a n A / B$ mediating resistance to methicillin or vancomycin in Gram-positive cocci, for example. We analyzed one plasma sample from a 59-year-old woman that suffered from sepsis following liver transplantation. Sequencing the corresponding plasma cfDNA at high depth (Additional file 13: Table S4) showed even coverage of the E. faecium genome (Fig. 4a; Additional file 14: Figure S9) and revealed unambiguous hits for resistance genes in the CARD database, including $v a n B, v_{a n} S_{B}$, tet1, and sat4 (Fig. 4b). Remarkably, our findings match with microbiological data showing that the infectious organism was E. faecium exhibiting vancomycin and tetracycline resistance (VRE), among others. Thus, assuming a sufficient sequence coverage, identification of genes conferring resistance using an unbiased highthroughput sequencing approach seems to be possible in principle.

\section{Discussion}

In recent years, several reports have been published on the use of metagenomics for the identification of viruses or microbes in patient samples. Most of these reports describe the identification of different viruses, including influenza A, norovirus, Ebola, arenaviruses, or emerging viruses from various specimens like nasopharyngeal swabs, serum, or solid tissue [23-29]. In addition, metagenomics, amongst other methods, has also been applied for identification of bacteria from urine, vaginal swabs, or sputum [30-33]. However, to the best of our knowledge, we report here the first proof-of-concept for quantitative NGS-based diagnosis of septicemia using plasma samples from septic patients and uninfected controls based on free circulating DNA. Several attempts have been made to use cfDNA quantification as a diagnostic and prognostic marker for sepsis outcome [34-36]. However, elevated cfDNA levels resulting from apoptotic or necrotic processes have also been described in other physiologic or pathologic conditions, including pregnancy, exercise, inflammatory diseases, trauma due to stroke, cancer, and surgery. Consequently, it is controversial whether elevated levels of circulating cfDNA are of diagnostic and prognostic value per se [36, 37]. Five out of seven septic patients (S9, S10, S19, S23, S60) underwent major abdominal surgery within 2 days of sepsis onset, which likely contributed to elevated cfDNA concentrations. However, septic patients showed a significantly higher share of cfDNA that could be assigned to microbial origin, suggesting a direct release of DNA from bacteria or co-release from phagocytic cells. This cfDNA could originate from both live and dead pathogens. However, as cfDNA is subject to considerable turnover, with a half-life in the range of minutes [38, 39], and obtained SIQ scores are very dynamic during the time course of infection, this suggests that cfDNA levels from microbial origin seem to precisely reflect the dynamics of infection, irrespective of whether cfDNA is released by dead or living cells. Due to the very short half-life of cfDNA, we therefore would also not expect to face a significant false positive rate. However, as our study only comprises a limited number of 
A

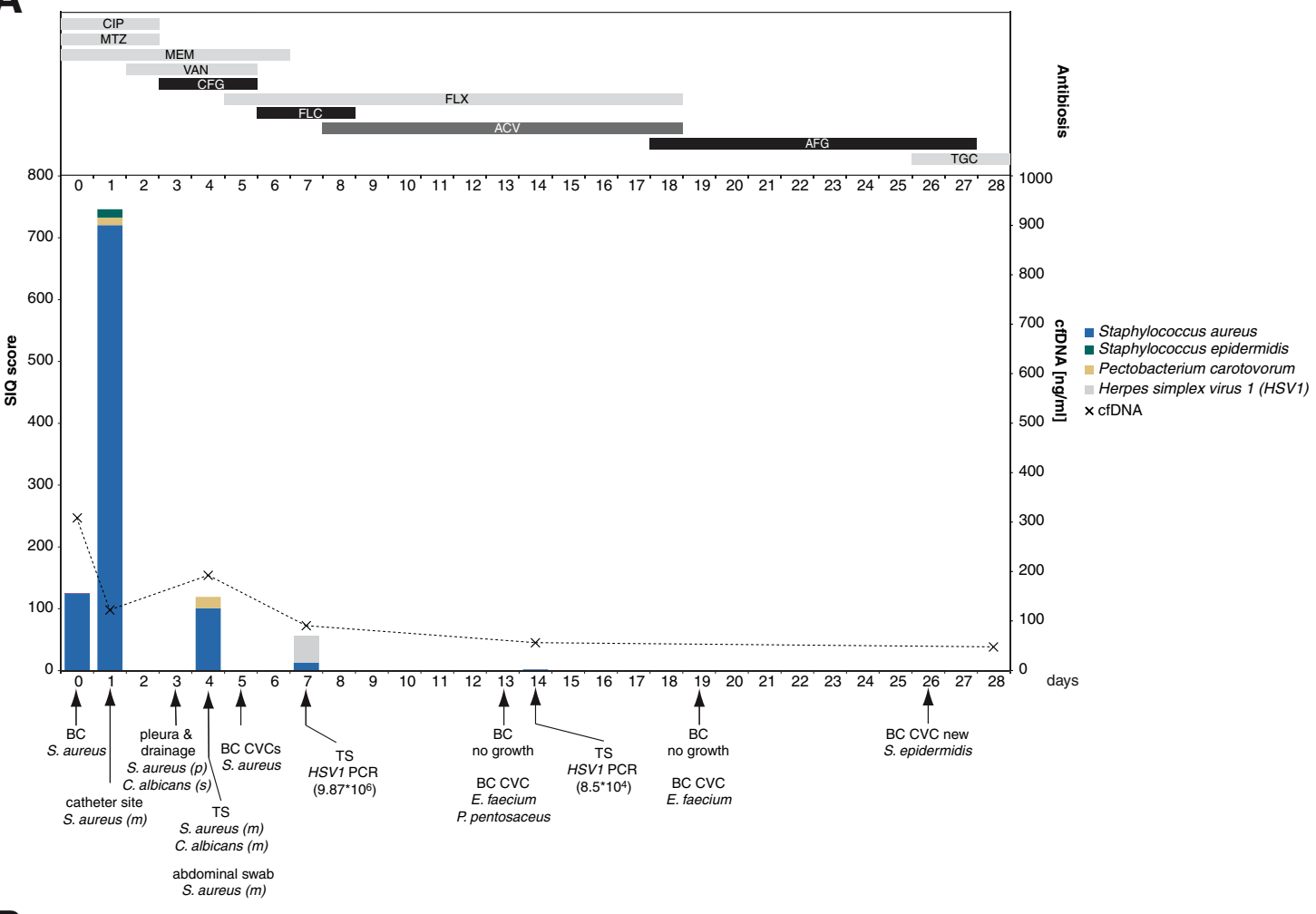

B

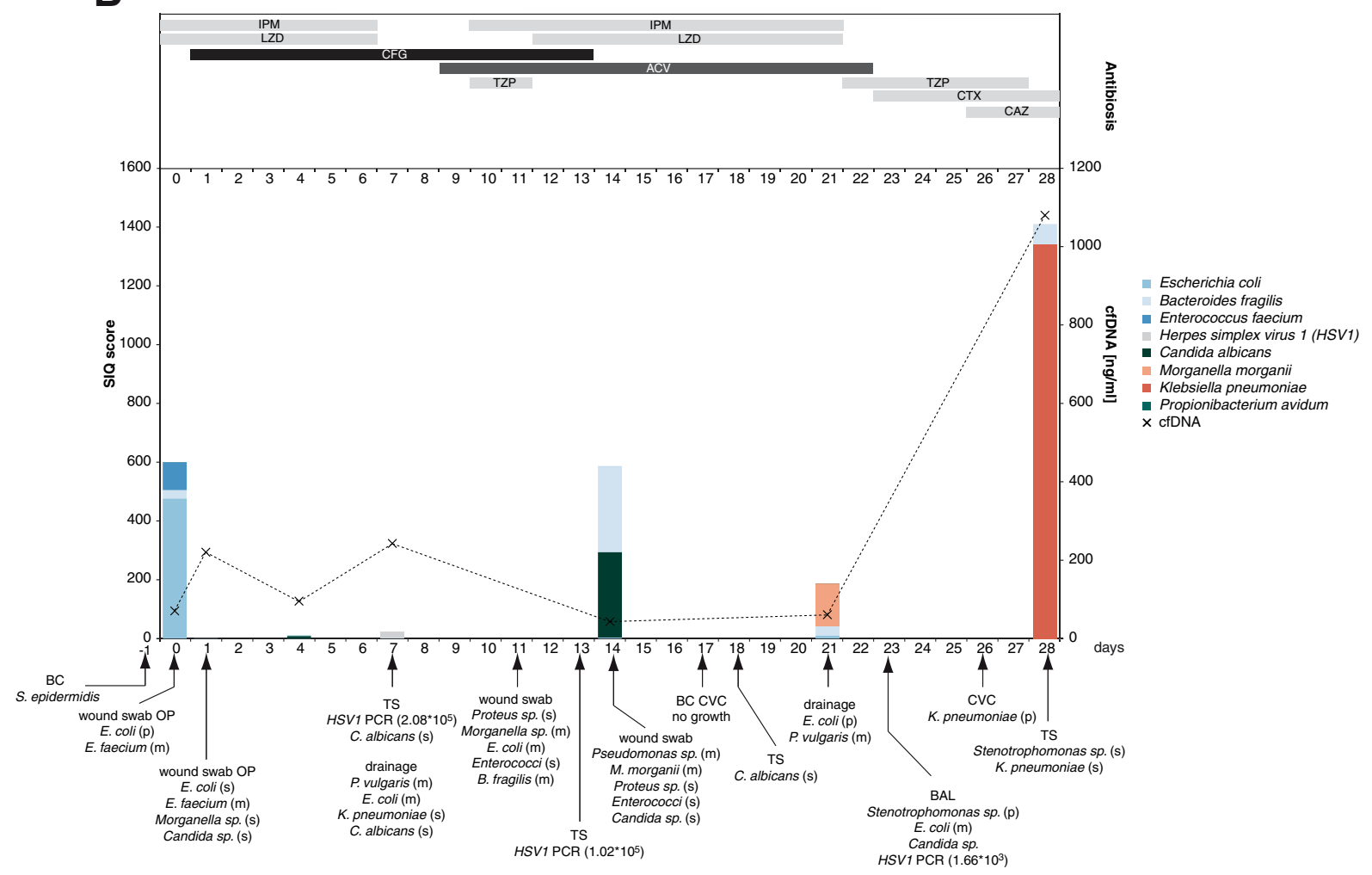

Fig. 3 (See legend on next page.) 
(See figure on previous page.)

Fig. 3 Time course SIQ analyses compared with conventional clinical microbiology data for two patients. a Time course of patient S10. A 68-yearold male patient presented with a tumor of his stomach with the need for a gastrectomy. Following the surgical procedure the patient suffered from septic shock due to severe pneumonia without any evidence of an anastomosis insufficiency. Staphylococcus aureus was shown to be the dominant organism in different secretions (e.g., tracheal secretion, abdominal wound swab, blood culture, etc.). In addition, pneumonia was shown to be accompanied (respectively boosted) by reactivation of herpes simplex virus type 1 (HSV1) in tracheal secretions. Following a prolonged weaning phase, the patient was then able to be discharged to the normal ward 6 weeks after the onset of septic shock. In this figure, the antibiotic treatment regime, SIQ scores for species identified via NGS/SEPseq, and cfDNA concentrations of the respective plasma samples are plotted for the trial period of 28 days. Pertinent (clinical microbiology) laboratory results are marked using arrows to indicate the day the clinical specimen was obtained. Abbreviations: BC blood culture, CVC central venous catheter, TS tracheal secretion, HSV herpes simplex virus, CIP ciproflocaxine, MTZ metronidazole, MEM meropenem, VAN vancomycin, CFG caspofungin, FLX flucloxacillin, FLC fluconazole, ACV aciclovir, AFG anidulafungin, TGC tigecycline. Anti-infectives are displayed as antibacterial antibiotics, antimycotics, and antivirals in light grey, black, and dark grey, respectively. The relative amount of bacteria found by conventional clinical microbiology is indicated with plenty $(p)$, medium $(m)$, or scarce $(s)$. (For a detailed list of the anti-infective abbreviations, see Table S5.) b Time course of patient S60. Following a complicated course of perforated sigmoid diverticulitis, a 70-year-old female patient presented for reconstruction of bowel continuity. In the postoperative phase the patient developed septic shock due to bowel leakage with the need for surgical revision. Abdominal wound swabs were shown to be positive for Escherichia coli and Enterococcus faecium. One day later the patient suffered from a second septic hit due to perforation of the colon with the need for surgical colectomy and construction of a stump by Hartmann. Afterwards the patient suffered from another septic hit due to an insufficiency of the stump by Hartmann. Accordingly, one further explorative laparotomy was performed and an intensive abdominal lavage was initiated. In the further course of the septic disease the patient developed a fourth septic hit due to ventilator-associated pneumonia triggered by E. coli, Stenotrophomonas maltophilia, and Klebsiella pneumoniae. Following a prolonged weaning phase the patient was then able to be transferred to the intermediate care ward after 3 months of ICU treatment. Ultimately, the patient could be discharged from hospital another 2 weeks later. Pertinent (clinical microbiology) laboratory results are marked using arrows to indicate the day the clinical specimen was obtained. Abbreviations: BC blood culture, CVC central venous catheter, TS tracheal secretion, BAL bronchoalveolar lavage, HSVI herpes simplex virus 1, IPM imipenem, LZD linezolid, CFG caspofungin, ACV aciclovir, TZP piperacillin-tazobactam, CTX cotrimoxazol, CAZ ceftazidime. Antibacterial antibiotics are colored in light grey. The relative amount of bacteria found by conventional clinical microbiology is indicated with plenty $(p)$, medium $(m)$, or scarce $(s)$. (For a detailed list of the anti-infective abbreviations, see Additional file 9: Table S5)

cases, further research and more comprehensive cohorts are needed to elucidate the source and mechanism of cfDNA release and its impact on false positive rates.

In patients suffering from severe sepsis or septic shock, positive blood cultures are obtained in only a fraction of cases despite a proven underlying rate of bacterial infection of $33 \%$ [40-42]. This is partially attributable to technical shortfalls in blood culture acquisition but is also due to fastidious organisms or very low rates of viable microorganisms in the blood stream [43]. A molecular approach with higher sensitivity for sepsis might, therefore, overcome the aforementioned limitations of classic microbiological diagnostics. However, state of the art molecular approaches based on PCR amplification of target sequences suffer from limited power to discriminate between contaminations, colonization, and infections,

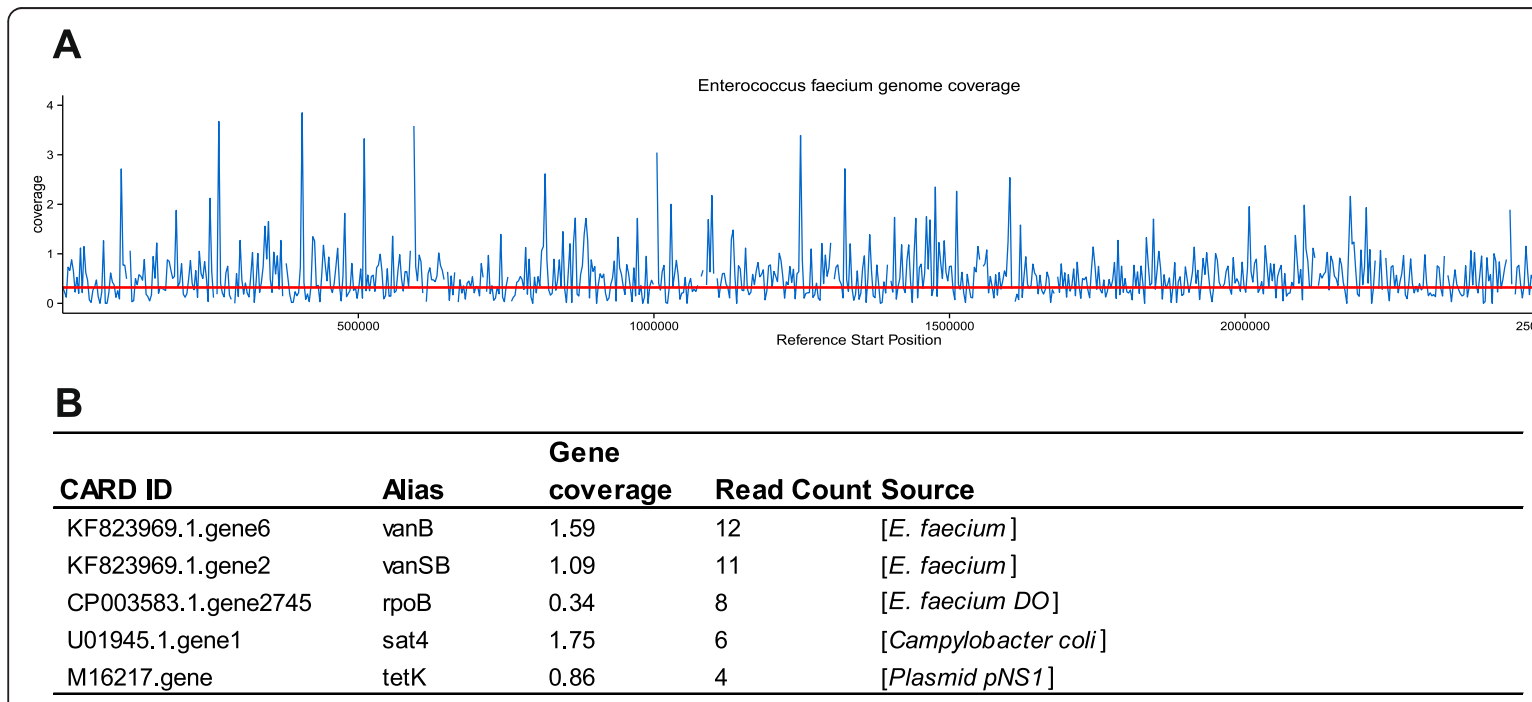

Fig. 4 Genome coverage and resistance profile of a patient infected with vancomycin-resistant E. faecium (VRE). a Mean genome coverage of approximately 0.3 of the $E$. faecium genome (2.8 Mb). $\mathbf{b}$ Table with hits to the CARD database. The CARD/GenBank accession number is listed as well as the alias gene name, gene coverage calculated from read length ratio to gene length, number of reads mapped to this gene, and the respective organism to which the sequence is assigned 
often revealing ambiguous or invalid results. In this context, NGS-based diagnostic testing might offer several advantages over PCR assays: (i) NGS is an open platform, providing the opportunity to detect bacterial, fungal, and viral pathogens in a single assay; (ii) NGS is quantitative through counting of sequence reads and calculation of statistical significance; (iii) NGS is unbiased and untargeted so benefits from any DNA sequence information within patient specimens, potentially delivering higher sensitivity and specificity. However, although NGS is becoming increasingly important in clinical microbiology (e.g., for strain typing), only sporadic reports of NGS-analyzed clinical specimens have been published to date, including one actionable single-case report of NGS-based diagnosis of Leptospira from cerebrospinal fluid [44-49]. Another recent paper, by De Vlaminck and coworkers [50], reports the identification of predominantly viral pathogens in cfDNA from patients after lung transplantation. Nevertheless, we currently lack NGS-based diagnostic approaches that, by combining quantitative values with statistical significance, provide a measure for relevance. Especially in specimens like plasma, where only the lowest amounts of microbial DNA can be expected, the specificity is challenged by the detection of microbial contaminants from laboratory reagents, workflows, or specimen sampling [18, 51]. We therefore developed a SIQ score to discriminate signal reads from noise caused by contaminant or commensal species. Since the incidence of viral and fungal infections in sepsis is less frequent, the SIQ scores for fungi and viruses will generally be higher than those for bacteria (as seen in patient S23; Additional file 11: Figure S6) as they are also less often detected in control samples. Consequently, the robustness of the SIQ score will be increased by using the most complete database of relevant genomes and a control group large enough to capture even small variances in the microbiome of healthy individuals.

Using our current workflow, a good correlation of NGS results with blood culture and other patient specimens was observed for all patients except patient S60. Moreover, this agreement was observed not only for bacteria but also for viruses and fungi. For patient $\mathrm{S} 60$ we found E. coli and $E$. faecium in plasma, which is in good agreement with wound swab findings but in contrast to blood culture, where S. epidermidis was identified one day before. Clinical records suggest that this patient suffered from gut complications, indicating that the NGS-based approach might be more sensitive and specific than blood culture, which has to be systematically evaluated by further studies.

Importantly, we could also demonstrate the potential of quantitative NGS-based diagnosis to identify genes conferring antibiotic resistance in plasma cfDNA. We unambiguously identified from a septic patient that underwent liver transplantation a number of reads that match with $100 \%$ identity to $v a n B$ and $v a n S_{B}$, known to confer resistance to vancomycin. Indeed, we could identify VRE as the infecting strain as confirmed by microbiological testing. Meanwhile, we were also able to detect mecA from methicillin-resistant $S$. aureus in other patient plasma samples (data not shown), indicating that the identification of major resistance genes is feasible with our approach. However, the applicability of the CARD antibiotic resistance database for diagnostic purposes is currently limited to genes which, per se, confer resistance to antibiotics, whereas the heterogeneity of resistance mechanisms also comprises point mutations, gene expression changes, and posttranslational modifications, for example. Consequently, using the current setup of the database and workflows, it is possible to identify resistance mechanisms based on acquisition of specific resistance genes like $m e c A, v a n A / B$, KPC, etc. but not necessarily to detect all resistance mechanisms comprehensively. Furthermore, it should be noted that mapping of reads to resistance-related genes relies on high read coverage for the respective species. For routine detection of resistance genes, enrichment strategies and complementation with expression data might be helpful in elucidating additional resistance mechanisms.

Finally, using our workflow to quantitatively identify microbial and viral nucleic acids in patient plasma (Additional file 15: Figure S8), the current total turnaround time starting from plasma adds up to approximately $31 \mathrm{~h}$, where the most time-consuming step is sequencing, which was accomplished in rapid run mode on a HiSeq2500 within $16 \mathrm{~h}$. This is competitive with standard blood culture with a time to positivity of 24$120 \mathrm{~h}$, where automated blood culture systems are routinely incubated for 5 days [43]. However, recent developments in sequencing technologies (i.e., Oxford Nanopore's MinION device) indicate that it will be possible to sequence even larger amounts of DNA in shorter times in a bedside setup. Using this technology it has already been reported that strain identification of clinically relevant strains and the corresponding resistance profiles is possible in approximately $2 \mathrm{~h}$ [52]. Furthermore, the identification of resistance genes has been shown to be manageable in 2 to $12 \mathrm{~h}$, which makes a "same day" diagnosis possible [52]. Nanopore sequencing would, therefore, allow a diagnostic decision within 3$4 \mathrm{~h}$ after initial diagnosis of sepsis using our workflow, providing a reasonable timeframe for effective therapeutic intervention. It should also be mentioned that current sequencing costs are of considerable importance for diagnostics. Although costs per base are constantly dropping, diagnostic approaches based on NGS are still comparatively expensive. Therefore, we expect that such a diagnostic workflow will be in a cost range similar to that of noninvasive prenatal testing (NIPT) when it was introduced several years before. This might limit a 
corresponding approach for diagnosis in critically ill patients in the beginning, for whom overall costs per patient and day are relatively high. But as seen for NIPT, costs per assay might significantly drop in short order following a broader application of NGS in clinics, thus making such an approach conceivable also from a financial point of view.

\section{Conclusions}

This work shows for the first time that identification of viruses, bacteria, and fungi from cfDNA in plasma of septic patients not only is technically feasible but also provides a basis to differentiate the relevant infecting organisms by establishing a quantitative score. These characteristics go beyond state-of-the-art molecular approaches for the diagnosis of infecting organisms in septic specimens, which are not open but instead based on PCR amplification of defined targets and are, in most cases, just qualitative in nature. Therefore, this approach allows for an unbiased analysis of bloodstream infections, which might be especially useful for the diagnosis of cases where classic microbiological or molecular diagnostic approaches fail. However, although we think that our approach might be more sensitive and specific than state-of-the-art technologies, additional clinical trials will be needed to exactly define the sensitivity and specificity as well as the positive and negative predictive value as this case study was limited by the low number of patients.

\section{Additional files}

Additional file 1: Table S1. List of fungal reference genomes included in the kraken database to identify microbial reads in non-human reads. (XLSX $11 \mathrm{~kb}$ )

Additional file 2: Figure S1. Bioanalyzer profiles of cfDNA isolated from septic patients, non-infected (post-)surgery controls, and healthy volunteers. a Gel-like visualization of the cfDNA profiles run on the Agilent Bioanalyzer with a High Sensitivity DNA chip. b Corresponding electropherograms, where the two outermost peaks are internal size standards. (PDF $661 \mathrm{~kb}$ )

Additional file 3: Table S6. Total read counts per sample and species. (XLSX $57 \mathrm{~kb}$ )

Additional file 4: Figure S2. Distribution of species-specific normalized read counts in septic patients and controls for major pathogens in the sepsis setting. Red: septic patients, blue: controls (elective surgery (timepoint T0) and healthy volunteers). (PDF $19 \mathrm{~kb}$ )

Additional file 5: Figure S3. Distribution of species-specific normalized read counts in septic patients and controls for potential contaminant species. Red, septic patients; blue, controls (elective surgery (timepoint T0) and healthy volunteers). (PDF $18 \mathrm{~kb}$ )

Additional file 6: Table S2. List of contaminant species identified from water controls. (XLSX $10 \mathrm{~kb}$ )

Additional file 7: Table S3. Patient characteristics, sequencing statistics, including normalized germ read counts, and SIQ scores for the complete dataset. (XLSX $21 \mathrm{~kb})$
Additional file 8: Figure S4. SIQ plot for post-surgery patients P1 T1 and P2 T1. (PDF $72 \mathrm{~kb}$ )

Additional file 9: Table S5. Anti-infective abbreviations. (XLSX 9 kb) Additional file 10: Figure S5. Time course of patient S9. An 82-yearold male patient presented with a tumor of his bile duct with the need for an enlarged right-sided hemihepatectomy. Following the surgical procedure, the patient suffered from septic shock due to Ogilvie syndrome and a right-sided hemicolectomie had to be performed. Septic shock was paralleled by repetitive positive blood cultures with Enterobacter cloacae, which was shown to be the cause of ventilator-associated pneumonia 1 week after sepsis onset. Following antibiotic treatment, two different biotypes of E. cloacae could be observed, both fulfilling the criteria of being multidrug resistant. The patient died 9 weeks after the onset of septic shock. In this figure, the antibiotic treatment regime, SIQ scores for species identified via NGS, and cfDNA concentrations of the respective plasma samples are plotted over the timeline of the trial period for patient S9. Pertinent clinical microbiology laboratory results are marked using arrows to indicate the day the clinical specimen was obtained. Abbreviations: BC blood culture, CVC central venous catheter, TS tracheal secretion, NL non-lysing, CIP ciproflocaxine, MTZ metronidazole, IPM imipenem, LZD linezolid, FLC fluconazole, VAN vancomycin. Anti-infectives are displayed as antibacterial antibiotics, antimycotics, and antivirals in light grey, black, and dark grey, respectively. The relative amount of bacteria found by conventional clinical microbiology is indicated with plenty $(p)$, medium $(m)$, or scarce (s). (For a detailed list of the anti-infective abbreviations, see Additional file 9: Table S5.) (PDF 16 kb)

Additional file 11: Figure S6. Time course of patient S11. A 62-year-old male patient presented with a multilocular hepatocellular carcinoma with the need for a left-sided hemihepatectomy. Following the surgical procedure the patient suffered from septic shock due to severe pneumonia with Klebsiella pneumoniae as the dominant organism in blood cultures as well as tracheal secretions. Empiric antibiotic therapy was performed with imipenem, which was then switched to moxifloxacin based on the susceptibility findings. In the further course of the disease, K. pneumoniae was shown to be multidrug resistant. Although antibiotic therapy was adapted according to the findings of susceptibility testing, the pulmonary septic focus could not be removed sufficiently. In the end, the patient died from ongoing septic shock due to pneumonia with $K$. pneumonia 2 months after study inclusion. In addition, septic disease was shown to be accompanied by a reactivation of herpes simplex virus type 1 (HSV1) as well as cytomegalovirus (CMV) in different secretions as assessed by a PCR-based diagnostic procedure. These findings were in good agreement with next generation sequencing (NGS) of plasma. In this figure, the antibiotic treatment regime, SIQ scores for species identified via NGS, and cfDNA concentrations of the respective plasma samples are plotted over the timeline of the trial period for patient S11. Pertinent (clinical microbiology) laboratory results are marked using arrows to indicate the day the clinical specimen was obtained. Abbreviations: $B C$ blood culture, CVC central venous catheter, TS tracheal secretion, GNST Gram-negative staphylococci, HSV1 herpes simplex virus 1, IPM imipenem, VAN vancomycin, MXF moxiflocaxin, CIP ciprofloxacin, TGC tigecycline, CAZ ceftazidime. Antibacterial antibiotics are displayed in light grey. The relative amount of bacteria found by conventional clinical microbiology is indicated with plenty $(p)$, medium $(m)$, or scarce $(s)$. (For a detailed list of the anti-infective abbreviations, see Additional file 9: Table S5) (PDF $16 \mathrm{~kb}$ )

Additional file 12: Figure S7. Time course of patient S23. A 77-year-old male patient presented with septic shock due to an acute abdominal infection. An ischemic colitis with a perforation of the sigma and severe peritonitis was identified to be the septic focus, so the patient underwent surgical colectomy. Abdominal wound swabs as well as the corresponding blood cultures were shown to be positive for Enterococcus faecium 2 days before inclusion in the study cohort. Empiric antibiotic therapy with imipenem and linezolid was therefore proven to be appropriate. In addition, this patient also revealed a reactivation of herpes simplex virus type 1 (HSV1) in tracheal secretions. These PCR-based findings could also be confirmed by next generation sequencing (NGS) of plasma. The antibiotic treatment regime, SIQ scores for species identified via NGS, and cfDNA concentrations of the respective plasma samples are plotted over the timeline of the trial period for patient S23. Pertinent (clinical 
microbiology) laboratory results are marked using arrows to indicate the day the clinical specimen was obtained. Abbreviations: $B C$ blood culture, TS tracheal secretion, CoNST coagulase negative staphylococci, IMP imipeneme, LZD linezolid, TZP tazobactam, CLI clindamycin, FLC fluconazole. Anti-infectives are colored as antibacterial antibiotics and antimycotics in light grey and black, respectively. The relative amount of bacteria found by conventional clinical microbiology is indicated with plenty $(p)$, medium $(m)$, or scarce (s). (For a detailed list of the anti-infective abbreviations, see Additional file 9: Table S5.) (PDF $15 \mathrm{~kb}$ )

Additional file 13: Table S4. Sequencing characteristics of the resistancecharacterized patient sample. (XLSX 8 kb)

Additional file 14: Figure S9. Mapping of Enterococcus faecium identified reads on the genome of Enterococcus faecium DO (NC_017960.1) using different minimum identity values while mapping. a Minimum identity of $65 \%$; b minimum identity of $70 \%$; c minimum identity of $85 \%$; d minimum identity of $90 \%$; e minimum identity of $95 \%$; $f$ minimum identity of $100 \%$. (PDF $382 \mathrm{~kb}$ )

Additional file 15: Figure S8. Workflow and time distribution of NGS-based pathogen identification and blood culture. Flow chart and bar chart of the individual steps and the time required for the NGS-based identification of bacteremia-causing species and conventional blood culture. As time to positivity varies substantially, a time frame of 24 to $120 \mathrm{~h}$ is given for blood culture. (PDF $42 \mathrm{~kb}$ )

\section{Abbreviations}

cfDNA, cell-free DNA; HSV, herpes simplex virus; NGS, next generation sequencing; qPCR, quantitative $P C R$; $S I Q$, sepsis indicating quantifier; VRE, vancomycin-resistant E. faecium.

\section{Acknowledgements}

We thank all patients and referring physicians and study nurses who submitted samples. We also acknowledge Karolina Glanz and Ute Krauser for excellent technical support.

\section{Funding}

This study was supported by the Fraunhofer Future Foundation. The Fraunhofer society had no role in study design, collection, analysis, or interpretation of data.

\section{Availability of data and materials}

The data supporting the conclusions of this article are available in the European Nucleotide Archive (ENA) repository under accession PRJEB13247 (http://www.ebi.ac.uk/ena/data/view/PRJEB13247).

\section{Authors' contributions}

SG, PS, SOD, SH, TB, MAW, and KS designed the study. SG, PS, CG, AvH, and KS acquired, analyzed, or interpreted the data. SG, PS, CG, TB, and KS wrote the manuscript. All authors critically revised the manuscript for important intellectual content and approved the final manuscript. PS and $\mathrm{AvH}$ provided bioinformatical data processing and analyses. Funding was obtained by MAW and KS. Further administrative, technical, or material support was provided by SG, PS, CG, SOD, SH, TB, and KS. The whole study was supervised by SG, MAW, AvH, and KS.

\section{Competing interests}

$\mathrm{SG}, \mathrm{PS}, \mathrm{AvH}$, and $\mathrm{KS}$ are co-inventors of a pending international patent on diagnostic algorithms. CG, SOD, MAW, SH, and TB declare that they have no competing interests.

\section{Ethics approval and consent to participate}

Human data result from a secondary analysis of a subset of patients participating in the RAMMSES trial (German Clinical Trials Register DRKS00000505). An amendment to the existing approval (Trial-Code-Nr. S123-2009) was written and approved by the Heidelberg ethics committee.

\section{Author details}

${ }^{1}$ Fraunhofer IGB, Nobelstr. 12, 70569 Stuttgart, Germany. ${ }^{2}$ IGVP, University of Stuttgart, Nobelstr. 12, 70569 Stuttgart, Germany. ${ }^{3}$ Department of Anesthesiology, Heidelberg University Hospital, Im Neuenheimer Feld 110 , 69120 Heidelberg, Germany. ${ }^{4}$ Center for Integrative Bioinformatics Vienna,
Max F. Perutz Laboratories, University of Vienna, Medical University of Vienna, Vienna, Austria. ${ }^{5}$ Bioinformatics and Computational Biology, Faculty of Computer Science, University of Vienna, Vienna, Austria.

Received: 27 January 2016 Accepted: 10 June 2016

Published online: 01 July 2016

\section{References}

1. Mayr FB, Yende S, Angus DC. Epidemiology of severe sepsis. Virulence. 2014;5(1):4-11.

2. Walkey AJ, Wiener RS, Lindenauer PK. Utilization patterns and outcomes associated with central venous catheter in septic shock: a population-based study. Crit Care Med. 2013;41(6):1450-7.

3. Dellinger RP, Levy MM, Rhodes A, Annane D, Gerlach H, Opal SM, Sevransky JE, Sprung CL, Douglas IS, Jaeschke R, et al. Surviving sepsis campaign: international guidelines for management of severe sepsis and septic shock: 2012. Crit Care Med. 2013;41(2):580-637.

4. Vincent JL, Brealey D, Libert N, Abidi NE, O'Dwyer M, Zacharowski K, Mikaszewska-Sokolewicz M, Schrenzel J, Simon F, Wilks M, et al. Rapid diagnosis of infection in the critically ill, a multicenter study of molecular detection in bloodstream infections, pneumonia, and sterile site infections. Crit Care Med. 2015:43(11):2283-91.

5. Bacconi A, Richmond GS, Baroldi MA, Laffler TG, Blyn LB, Carolan HE, Frinder MR, Toleno DM, Metzgar D, Gutierrez JR, et al. Improved sensitivity for molecular detection of bacterial and Candida infections in blood. J Clin Microbiol. 2014;52(9):3164-74.

6. Ecker DJ, Sampath R, Li H, Massire C, Matthews HE, Toleno D, Hall TA, Blyn LB, Eshoo MW, Ranken R, et al. New technology for rapid molecular diagnosis of bloodstream infections. Expert Rev Mol Diagn. 2010:10(4):399-415.

7. Eshoo MW, Crowder CD, Li H, Matthews HE, Meng S, Sefers SE, Sampath R, Stratton CW, Blyn LB, Ecker DJ, et al. Detection and identification of Ehrlichia species in blood by use of PCR and electrospray ionization mass spectrometry. J Clin Microbiol. 2010;48(2):472-8.

8. Kaleta EJ, Clark AE, Cherkaoui A, Wysocki VH, Ingram EL, Schrenzel J, Wolk DM. Comparative analysis of PCR-electrospray ionization/mass spectrometry (MS) and MALDI-TOF/MS for the identification of bacteria and yeast from positive blood culture bottles. Clin Chem. 2011;57(7):1057-67.

9. Kaleta EJ, Clark AE, Johnson DR, Gamage DC, Wysocki VH, Cherkaoui A, Schrenzel J, Wolk DM. Use of PCR coupled with electrospray ionization mass spectrometry for rapid identification of bacterial and yeast bloodstream pathogens from blood culture bottles. J Clin Microbiol. 2011;49(1):345-53.

10. Brenner T, Fleming T, Uhle F, Silaff $S$, Schmitt F, Salgado E, Ulrich A, Zimmermann S, Bruckner T, Martin E, et al. Methylglyoxal as a new biomarker in patients with septic shock: an observational clinical study. Crit Care. 2014;18(6):683.

11. Levy MM, Fink MP, Marshall JC, Abraham E, Angus D, Cook D, Cohen J, Opal SM, Vincent JL, Ramsay G. 2001 SCCM/ESICM/ACCP/ATS/SIS International Sepsis Definitions Conference. Crit Care Med. 2003;31(4):1250-6.

12. Calandra T, Cohen J. The international sepsis forum consensus conference on definitions of infection in the intensive care unit. Crit Care Med. 2005;33(7):1538-48

13. Gumbinger C, Hug A, Murle B, Berger B, Zorn M, Becker KP, Zimmermann S, Dalpke AH, Veltkamp R. Early blood-based microbiological testing is ineffective in severe stroke patients. J Neurol Sci. 2013;325(1-2):46-50.

14. Brenner T, Rosenhagen C, Hornig I, Schmidt K, Lichtenstern C, Mieth M, Bruckner T, Martin E, Schnitzler P, Hofer S, et al. Viral infections in septic shock (VISS-trial)-crosslinks between inflammation and immunosuppression. J Surg Res. 2012;176(2):571-82.

15. Mischnik A, Mieth M, Busch CJ, Hofer S, Zimmermann S. First evaluation of automated specimen inoculation for wound swab samples by use of the Previ Isola system compared to manual inoculation in a routine laboratory: finding a cost-effective and accurate approach. J Clin Microbiol. 2012;50(8):2732-6

16. Mischnik A, Trampe M, Zimmermann S. Evaluation of the impact of automated specimen inoculation, using Previ Isola, on the quality of and technical time for stool cultures. Ann Lab Med. 2015;35(1):82-8.

17. Horie M, Honda T, Suzuki Y, Kobayashi $Y$, Daito T, Oshida T, Ikuta K, Jern P, Gojobori T, Coffin JM, et al. Endogenous non-retroviral RNA virus elements in mammalian genomes. Nature. 2010;463(7277):84-7. 
18. Salter SJ, Cox MJ, Turek EM, Calus ST, Cookson WO, Moffatt MF, Turner P, Parkhill J, Loman NJ, Walker AW. Reagent and laboratory contamination can critically impact sequence-based microbiome analyses. BMC Biol. 2014;12:87.

19. Giacona MB, Ruben GC, Iczkowski KA, Roos TB, Porter DM, Sorenson GD. Cell-free DNA in human blood plasma: length measurements in patients with pancreatic cancer and healthy controls. Pancreas. 1998;17(1):89-97.

20. Lichtenstein AV, Melkonyan HS, Tomei LD, Umansky SR. Circulating nucleic acids and apoptosis. Ann N Y Acad Sci. 2001;945:239-49.

21. Martins GA, Kawamura MT, Carvalho MG. Detection of DNA in the plasma of septic patients. Ann N Y Acad Sci. 2000;906:134-40.

22. Zeerleder S, Zwart B, Wuillemin WA, Aarden LA, Groeneveld AB, Caliezi C, van Nieuwenhuijze AE, van Mierlo GJ, Eerenberg AJ, Lammle B, et al. Elevated nucleosome levels in systemic inflammation and sepsis. Crit Care Med. 2003:31(7):1947-51

23. Yang J, Yang F, Ren L, Xiong Z, Wu Z, Dong J, Sun L, Zhang T, Hu Y, Du J, et al. Unbiased parallel detection of viral pathogens in clinical samples by use of a metagenomic approach. J Clin Microbiol. 2011;49(10):3463-9.

24. Towner JS, Sealy TK, Khristova ML, Albarino CG, Conlan S, Reeder SA, Quan PL, Lipkin WI, Downing R, Tappero JW, et al. Newly discovered ebola virus associated with hemorrhagic fever outbreak in Uganda. PLoS Pathog. 2008;4(11):e1000212.

25. Palacios G, Druce J, Du L, Tran T, Birch C, Briese T, Conlan S, Quan PL, Hui J, Marshall J, et al. A new arenavirus in a cluster of fatal transplant-associated diseases. N Engl J Med. 2008;358(10):991-8.

26. Nakamura S, Yang CS, Sakon N, Ueda M, Tougan T, Yamashita A, Goto N, Takahashi K, Yasunaga T, Ikuta K, et al. Direct metagenomic detection of viral pathogens in nasal and fecal specimens using an unbiased high-throughput sequencing approach. PLoS One. 2009;4(1):e4219.

27. Kohl C, Brinkmann A, Dabrowski PW, Radonic A, Nitsche A, Kurth A. Protocol for metagenomic virus detection in clinical specimens. Emerg Infect Dis. 2015:21(1):48-57.

28. Greninger AL, Chen EC, Sittler T, Scheinerman A, Roubinian N, Yu G, Kim E, Pillai DR, Guyard C, Mazzulli T, et al. A metagenomic analysis of pandemic influenza A (2009 H1N1) infection in patients from North America. PLoS One. 2010:5(10):e13381.

29. Briese T, Paweska JT, McMullan LK, Hutchison SK, Street C, Palacios G, Khristova ML, Weyer J, Swanepoel R, Egholm M, et al. Genetic detection and characterization of Lujo virus, a new hemorrhagic fever-associated arenavirus from southern Africa. PLoS Pathog. 2009:5(5):e1000455.

30. Seth-Smith HM, Harris SR, Skilton RJ, Radebe FM, Golparian D, Shipitsyna E, Duy PT, Scott P, Cutcliffe LT, O'Neill C, et al. Whole-genome sequences of Chlamydia trachomatis directly from clinical samples without culture. Genome Res. 2013;23(5):855-66.

31. Doughty EL, Sergeant MJ, Adetifa I, Antonio M, Pallen MJ. Culture-independent detection and characterisation of Mycobacterium tuberculosis and $\mathrm{M}$. africanum in sputum samples using shotgun metagenomics on a benchtop sequencer. PeerJ. 2014;2:e585.

32. Andersson $P$, Klein M, Lilliebridge RA, Giffard PM. Sequences of multiple bacterial genomes and a Chlamydia trachomatis genotype from direct sequencing of DNA derived from a vaginal swab diagnostic specimen. Clin Microbiol Infect. 2013;19(9):E405-8.

33. Hasman H, Saputra D, Sicheritz-Ponten $T$, Lund O, Svendsen CA, Frimodt-Moller N, Aarestrup FM. Rapid whole-genome sequencing for detection and characterization of microorganisms directly from clinical samples. J Clin Microbiol. 2014;52(1):139-46.

34. Avriel A, Paryente Wiessman M, Almog Y, Perl Y, Novack V, Galante O, Klein M, Pencina MJ, Douvdevani A. Admission cell free DNA levels predict 28-day mortality in patients with severe sepsis in intensive care. PLoS One. 2014;9(6):e100514.

35. Dwivedi DJ, Toltl LJ, Swystun LL, Pogue J, Liaw KL, Weitz Jl, Cook DJ, Fox-Robichaud AE, Liaw PC, Canadian Critical Care Translational Biology G. Prognostic utility and characterization of cell-free DNA in patients with severe sepsis. Crit Care. 2012;16(4):R151.

36. Rhodes A, Wort SJ, Thomas H, Collinson P, Bennett ED. Plasma DNA concentration as a predictor of mortality and sepsis in critically ill patients. Crit Care. 2006;10(2):R60.

37. Garnacho-Montero J, Huici-Moreno MJ, Gutierrez-Pizarraya A, Lopez I, Marquez-Vacaro JA, Macher H, Guerrero JM, Puppo-Moreno A. Prognostic and diagnostic value of eosinopenia, C-reactive protein, procalcitonin, and circulating cell-free DNA in critically ill patients admitted with suspicion of sepsis. Crit Care. 2014;18(3):R116.

38. Rumore P, Muralidhar B, Lin M, Lai C, Steinman CR. Haemodialysis as a model for studying endogenous plasma DNA: oligonucleosome-like structure and clearance. Clin Exp Immunol. 1992;90(1):56-62.

39. Zhang J, Tong KL, Li PK, Chan AY, Yeung CK, Pang CC, Wong TY, Lee KC, Lo YM. Presence of donor- and recipient-derived DNA in cell-free urine samples of renal transplantation recipients: urinary DNA chimerism. Clin Chem. 1999:45(10):1741-6.

40. Brunkhorst FM, Oppert M, Marx G, Bloos F, Ludewig K, Putensen C, Nierhaus A, Jaschinski U, Meier-Hellmann A, Weyland A, et al. Effect of empirical treatment with moxifloxacin and meropenem vs meropenem on sepsis-related organ dysfunction in patients with severe sepsis: a randomized trial. JAMA. 2012;307(22):2390-9.

41. Engel $\mathrm{C}$, Brunkhorst FM, Bone HG, Brunkhorst $\mathrm{R}$, Gerlach $\mathrm{H}$, Grond $\mathrm{S}$, Gruendling M, Huhle G, Jaschinski U, John S, et al. Epidemiology of sepsis in Germany: results from a national prospective multicenter study. Intensive Care Med. 2007;33(4):606-18.

42. Schmitz RP, Keller PM, Baier M, Hagel S, Pletz MW, Brunkhorst FM. Quality of blood culture testing-a survey in intensive care units and microbiological laboratories across four European countries. Crit Care. 2013;17(5):R248.

43. Kirn TJ, Weinstein MP. Update on blood cultures: how to obtain, process, report, and interpret. Clin Microbiol Infect. 2013;19(6):513-20.

44. Be NA, Allen JE, Brown TS, Gardner SN, McLoughlin KS, Forsberg JA, Kirkup BC, Chromy BA, Luciw PA, Elster EA, et al. Microbial profiling of combat wound infection through detection microarray and next-generation sequencing. J Clin Microbiol. 2014;52(7):2583-94.

45. Brown JR, Morfopoulou S, Hubb J, Emmett WA, Ip W, Shah D, Brooks T, Paine SM, Anderson G, Virasami A, et al. Astrovirus VA1/HMO-C: an increasingly recognized neurotropic pathogen in immunocompromised patients. Clin Infect Dis. 2015;60(6):881-8.

46. Kommedal O, Wilhelmsen MT, Skrede S, Meisal R, Jakovljev A, Gaustad P, Hermansen NO, Vik-Mo E, Solheim O, Ambur OH, et al. Massive parallel sequencing provides new perspectives on bacterial brain abscesses. J Clin Microbiol. 2014;52(6):1990-7.

47. Naccache SN, Peggs KS, Mattes FM, Phadke R, Garson JA, Grant P, Samayoa E, Federman S, Miller S, Lunn MP, et al. Diagnosis of neuroinvasive astrovirus infection in an immunocompromised adult with encephalitis by unbiased next-generation sequencing. Clin Infect Dis. 2015;60(6):919-23.

48. Wilson MR, Naccache SN, Samayoa E, Biagtan M, Bashir H, Yu G, Salamat SM, Somasekar S, Federman S, Miller S, et al. Actionable diagnosis of neuroleptospirosis by next-generation sequencing. N Engl J Med. 2014;370(25):2408-17.

49. Naccache SN, Federman S, Veeraraghavan N, Zaharia M, Lee D, Samayoa E, Bouquet J, Greninger AL, Luk KC, Enge B, et al. A cloud-compatible bioinformatics pipeline for ultrarapid pathogen identification from nextgeneration sequencing of clinical samples. Genome Res. 2014;24(7):1180-92.

50. De Vlaminck I, Martin L, Kertesz M, Patel K, Kowarsky M, Strehl C, Cohen G, Luikart H, Neff NF, Okamoto J, et al. Noninvasive monitoring of infection and rejection after lung transplantation. Proc Natl Acad Sci U S A. 2015;112(43):13336-41.

51. Strong MJ, Xu G, Morici L, Splinter Bon-Durant S, Baddoo M, Lin Z, Fewell C, Taylor CM, Flemington EK. Microbial contamination in next generation sequencing: implications for sequence-based analysis of clinical samples. PLoS Pathog. 2014;10(11):e1004437.

52. Cao MD, Ganesamoorthy D, Elliot AG, Zhang H, Cooper MA, Coin L. Real-time strain typing and analysis of antibiotic resistance potential using Nanopore MinION sequencing. bioRxiv. 2015. doi: http://dx.doi. org/10.1101/019356. 